\title{
First-Order Sideband Pulling in Atomic Frequency Standards
}

\author{
Jon H. Shirley, Thomas P. Heavner, and Steven R. Jefferts, Member, IEEE
}

\begin{abstract}
Many high-performance atomic frequency standards, including cesium fountains and most optical standards, operate with pulsed measurements, that is, an atomic sample is prepared for measurement, the measurement is made, and the process is repeated at fixed intervals. By introducing frequency modulation of the excitation coherent with the measurement cycle time, we have observed biases in our primary frequency standard NIST-F1 that are much larger than the biases predicted by previous theories. We derive a theory of such biases for both Rabi and Ramsey excitations. We find agreement with the experimental measurements.
\end{abstract}

Index Terms-Atomic clock, cesium fountain, primary frequency standard.

\section{INTRODUCTION}

$\mathbf{M}$ ANY frequency standards operate in a pulsed measurement regime. That is, an atomic sample is prepared for measurement, the measurement is made, and the process is repeated after a fixed cycle period. Cesium fountain primary frequency standards [1] and many optical standards [2] operate in this fashion. The pulsed nature of the measurement process can reveal effects that are absent or highly suppressed in continuous measurements, such as those made with thermal-beam frequency standards. Here, we consider the first-order effect of sidebands on the exciting radiation when the sideband offset frequency is coherent with the measurement cycle.

It has long been known that the presence of unbalanced sidebands on the exciting field can cause a shift of the resonance of a two-level system [3], [4]. Early work only considered the case of sidebands that are far removed from the carrier compared with the width of the Rabi resonance. Later, a result that is valid for any offset of the sideband was published, revealing the complex behavior of the shift as a function of the offset frequency [5]. These analyses computed shifts of second order in the sideband amplitude. The first-order shift was neglected because it depends on the initial phase of the sideband seen by atoms entering an excitation region. The continuous signals from atomic-beam frequency standards average over such initial phases. However, the pulsed operation of fountain

Manuscript received June 11, 2008; revised October 21, 2008. First published January 9, 2009; current version published March 10, 2009. The Associate Editor coordinating the review process for this paper was Dr. Tae-Weon Kang.

The authors are with the Time and Frequency Division, National Institute of Standards and Technology (NIST), Boulder, CO 80305 USA (e-mail: jefferts@ boulder.nist.gov).

Color versions of one or more of the figures in this paper are available online at http://ieeexplore.ieee.org.

Digital Object Identifier 10.1109/TIM.2008.2008590 standards introduces the possibility of phase coherence between the sidebands and the fountain cycle period [6].

We have deliberately introduced phase modulation of the excitation coherent with the cycle period of the cesium fountain primary frequency standard NIST-F1. We have observed sideband pulling shifts that are much larger than those predicted by the second-order theory [5]. The purpose of this paper is to present a theory for such shifts and experimental evidence for them. The Ramsey result of the theory, without derivation, was reported earlier [7], along with some experimental data.

We start by finding an approximate solution of the timedependent Schrödinger equation using the theoretical framework developed by Shirley et al. [8]. From this solution, we derive expressions for the frequency shifts when either a Rabi- or Ramsey-style excitation is used. Experimental data are then reported in agreement with the theory. Finally, we summarize the importance of these results.

\section{THEORY}

We begin with the Hamiltonian for a two-level system (cf. [8, eq. (7)] or [9, eq. (5)]) that is driven by exciting fields at two frequencies

$$
\mathcal{H}=\hbar\left(\begin{array}{cc}
\omega_{a} & M \\
M & \omega_{b}
\end{array}\right)
$$

where $M=2 b \cos \omega t+2 b_{1} \cos [(\omega+\Omega) t+\varphi]$.

Here, $\hbar \omega_{a}$ and $\hbar \omega_{b}$ are the energies of the upper and lower levels, respectively. The primary exciting field has a frequency $\omega$ and an amplitude that is represented by the Rabi frequency $2 b$. Similarly, the sideband has a frequency $\omega+\Omega$ and an amplitude that is represented by the Rabi frequency $2 b_{1}$. The sideband offset frequency $\Omega$ and amplitude $b_{1}$ are assumed to be small compared with $\omega$ and $b$, respectively. The phase of the sideband frequency at the time $t=0$ when atom excitation begins is denoted by $\varphi$. Both $b$ and $b_{1}$ may be time dependent but at rates that are small compared with the frequency $\omega$.

In the rotating-wave approximation, the Hamiltonian [see (1)] becomes $\left(\omega_{a}>\omega_{b}\right)$

$$
\mathcal{H}=\hbar\left(\begin{array}{cc}
\omega_{a} & b e^{-i \omega t}+b_{1} e^{-i(\omega+\Omega) t-i \varphi} \\
b e^{i \omega t}+b_{1} e^{i(\omega+\Omega) t+i \varphi} & \omega_{b}
\end{array}\right) .
$$

The complex conjugation of the off-diagonal elements retains the Hermiticity of the Hamiltonian. By using the "phasefactored" probability amplitudes, the rapid exponential time dependence of the off-diagonal elements in (2) can be transformed to a constant contribution to the diagonal elements 
(cf. [8, eqs. (9) and (10)]). The resulting time-dependent Schrödinger equation for the system is

$$
i \hbar \frac{d}{d t}\left(\begin{array}{l}
\alpha \\
\beta
\end{array}\right)=\hbar\left(\begin{array}{cc}
-\Delta & b+b_{1} e^{-i \Omega t-i \varphi} \\
b+b_{1} e^{i \Omega t+i \varphi} & \Delta
\end{array}\right)\left(\begin{array}{l}
\alpha \\
\beta
\end{array}\right) .
$$

Here, $\Delta$ is half of the detuning from the atomic resonance $\omega_{0}$ : $\Delta=(1 / 2)\left(\omega-\omega_{0}\right)$, where $\omega_{0}=\omega_{a}-\omega_{b}$ is the frequency separation of the levels. The quantities $\Delta, b$, and $b_{1}$ are all real. We impose the initial conditions $\alpha(0)=1$ and $\beta(0)=0$. Then, $\alpha$ is the probability amplitude that the system remains in its initial state, and $\beta$ is the probability amplitude that the system changes state.

The amplitudes $\alpha$ and $\beta$ are complex. If we write out the real and imaginary parts of the two equations in (3), we have four coupled differential equations in four real variables

$$
\begin{aligned}
d \operatorname{Re} \alpha / d t= & -\Delta \operatorname{Im} \alpha+\left[b+b_{1} \cos (\Omega t+\varphi)\right] \operatorname{Im} \beta \\
& -b_{1} \sin (\Omega t+\varphi) \operatorname{Re} \beta \\
d \operatorname{Im} \alpha / d t= & \Delta \operatorname{Re} \alpha-\left[b+b_{1} \cos (\Omega t+\varphi)\right] \operatorname{Re} \beta \\
& -b_{1} \sin (\Omega t+\varphi) \operatorname{Im} \beta \\
d \operatorname{Re} \beta / d t= & \Delta \operatorname{Im} \beta+\left[b+b_{1} \cos (\Omega t+\varphi)\right] \operatorname{Im} \alpha \\
& +b_{1} \sin (\Omega t+\varphi) \operatorname{Re} \alpha \\
d \operatorname{Im} \beta / d t= & -\Delta \operatorname{Re} \beta-\left[b+b_{1} \cos (\Omega t+\varphi)\right] \operatorname{Re} \alpha \\
& +b_{1} \sin (\Omega t+\varphi) \operatorname{Im} \alpha .
\end{aligned}
$$

When $b_{1}$ is zero, and the initial conditions for (3) hold, we see that $\operatorname{Re} \alpha$ and $\operatorname{Im} \beta$ will be even functions of the detuning $\Delta$, whereas $\operatorname{Im} \alpha$ and $\operatorname{Re} \beta$ will be odd functions. The transition probability, which is the sum of the squares of the real and imaginary parts of $\beta$, is thus an even function of detuning and, hence, is centered on the resonance. This remains true when the terms with the coefficient $b_{1} \cos (\Omega t+\varphi)$ are included. These latter terms, which represent the amplitude variations of $b$, cause a small symmetric change in the lineshape and, thus, no frequency shift.

The presence of the terms with the coefficient $b_{1} \sin (\Omega t+$ $\varphi)$ destroys this symmetry in detuning. These terms, which represent the phase changes in the exciting field, introduce antisymmetric changes in the lineshape, which cause frequency shifts. To first order in $b_{1}$, the effects of the terms in $b_{1} \cos (\Omega t+$ $\varphi)$ and $b_{1} \sin (\Omega t+\varphi)$ are independent. Hence, to find the firstorder shift, we only need to solve (4) and only without the terms in $b_{1} \cos (\Omega t+\varphi)$.

When the excitation amplitudes $b$ and $b_{1}$ are constant in time, (4) may be solved by transforming to a basis in which the Hamiltonian without the perturbation is diagonal. This is accomplished by the constant matrix

$$
A=\frac{1}{\sqrt{2 p}}\left(\begin{array}{cc}
\sqrt{p+\Delta} & -\sqrt{p-\Delta} \\
\sqrt{p-\Delta} & \sqrt{p+\Delta}
\end{array}\right)
$$

where $p=\sqrt{b^{2}+\Delta^{2}}$. We call the wave function amplitudes in the new basis $\xi$ and $\eta$. They obey the differential equations

$$
\begin{aligned}
& i d \xi / d t=-p \xi-i b_{1} \sin (\Omega t+\varphi) \eta \\
& i d \eta / d t=+p \eta+i b_{1} \sin (\Omega t+\varphi) \xi
\end{aligned}
$$

with the initial conditions

$$
\begin{aligned}
& \xi(0)=\sqrt{(p+\Delta) / 2 p} \\
& \eta(0)=\sqrt{(p-\Delta) / 2 p} .
\end{aligned}
$$

Let $\xi_{0}$ and $\eta_{0}$ be the solutions of (5) in the absence of $b_{1}$. The first-order solutions $\xi_{1}$ and $\eta_{1}$ of (5) are then found by inserting $\eta_{0}$ and $\xi_{0}$ into the $b_{1}$ terms. The result at time $\tau$ is

$$
\begin{aligned}
& \xi_{1}(\tau)=-\sqrt{(p-\Delta) / 2 p} F(\tau) \\
& \eta_{1}(\tau)=+\sqrt{(p+\Delta) / 2 p} F^{*}(\tau)
\end{aligned}
$$

where

$$
F(\tau)=\int_{0}^{\tau} b_{1} \sin (\Omega t+\varphi) \exp (i p \tau-2 i p t) d t
$$

When we transform back to the original basis, we find the usual amplitudes in the absence of $b_{1}$ (see [8, eq. (19)]) as

$$
\begin{aligned}
\alpha_{0}(\tau) & =\cos p \tau+i(\Delta / p) \sin p \tau \\
\beta_{0}(\tau) & =-i(b / p) \sin p \tau .
\end{aligned}
$$

The first-order corrections are

$$
\begin{aligned}
\alpha_{1}(\tau) & =-i(b / p) \operatorname{Im} F(\tau) \\
\beta_{1}(\tau) & =\operatorname{Re} F(\tau)-i(\Delta / p) \operatorname{Im} F(\tau)
\end{aligned}
$$

Equations (7)-(9) are the basic results needed for evaluating the sideband pulling shifts.

\section{A. Rabi Case}

Consider the excitation at a constant amplitude $b$ for a time $\tau$. The transition probability is simply given by

$$
\begin{aligned}
P & =\left|\beta^{2}\right| \approx\left|\beta_{0}^{2}\right|+2 \operatorname{Re}\left(\beta_{0}^{*} \beta_{1}\right) \\
& =\left(b^{2} / p^{2}\right) \sin ^{2} p \tau+\left(2 \Delta b / p^{2}\right) \sin p \tau \operatorname{Im} F(\tau)
\end{aligned}
$$

to first order in $b_{1}$. The second term in (10), which is odd in detuning, introduces a shift in the resonance. For slow square-wave modulation of the Rabi lineshape, the resonance condition is $P\left(-\omega_{m}+\delta \omega_{\mathrm{Rab}}\right)=P\left(\omega_{m}+\delta \omega_{\mathrm{Rab}}\right)$, where $\omega_{m}$ is the modulation amplitude, and $\delta \omega_{\mathrm{Rab}}$ is the shift due to the presence of the sideband. This resonance condition is achieved when

$$
\delta \omega_{\mathrm{Rab}}=\frac{-2 p_{m}^{2} \operatorname{Im} F_{m}(\tau)}{b\left(\sin p_{m} \tau-p_{m} \tau \cos p_{m} \tau\right)}
$$

with $p_{m}^{2}=b^{2}+\left(\omega_{m}^{2} / 4\right)$. The subscript $m$ on $F$ means that $p$ should be evaluated at $p_{m}$. The denominator in (11) is 


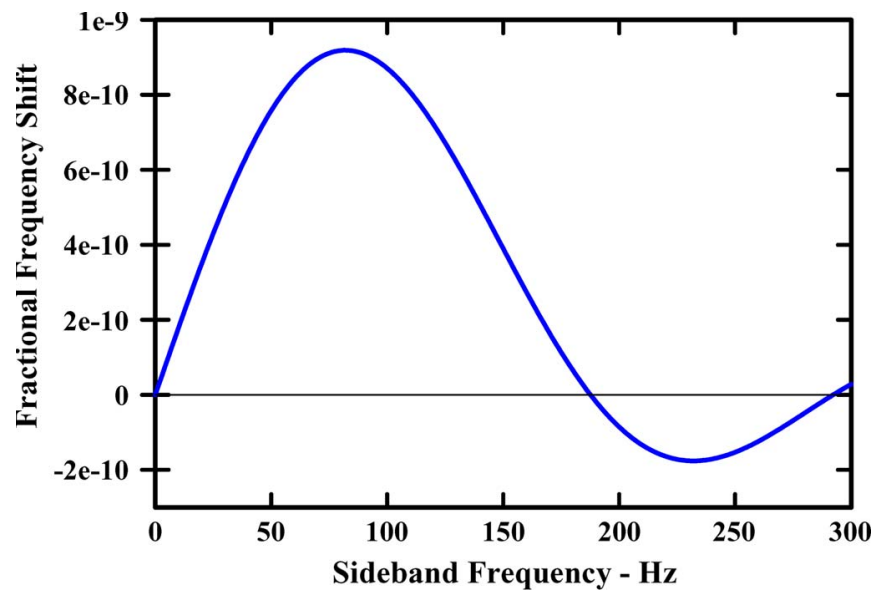

Fig. 1. Theoretical amplitude of the sideband pulling shift for Rabi excitation with phase modulation. The modulation amplitude was $6^{\circ}$, and the excitation amplitude was optimum. (Note that $6^{\circ}$ of phase corresponds to a sideband amplitude of $-20 \mathrm{dBc}$.)

proportional to the slope of the Rabi resonance curve at the detuning $\omega_{m}$.

With the aid of trigonometric identities, the integral in (7) can be analytically expressed as

$$
\begin{aligned}
& \operatorname{Im} F(\tau)=\frac{2 b_{1}}{\left(\Omega^{2}-4 p^{2}\right)}\left[\Omega \sin p \tau \cos \frac{1}{2} \Omega \tau-2 p \cos p \tau \sin \frac{1}{2} \Omega \tau\right] \\
& \times \cos \left(\frac{1}{2} \Omega \tau+\varphi\right) .
\end{aligned}
$$

The sideband amplitude $b_{1}$ has also been assumed constant during the excitation. Note that (12) is continuous at $\Omega= \pm 2 p$ since the numerator also vanishes there. Equation (12) then reduces to

$$
\operatorname{Im} F(\tau)= \pm \frac{b_{1}}{2 p}[\sin p \tau \cos p \tau-p \tau] \cos (p \tau \pm \varphi)
$$

For a small offset $(\Omega \tau \ll 1, \Omega \ll b)$, the shift reduces to

$$
\delta \omega_{\mathrm{Rab}}=\left(b_{1} / b\right) \Omega \cos \varphi
$$

which is just the instantaneous frequency of the phase modulation.

From (12), the shift is sinusoidal in the initial phase $\varphi$. Averaging over all the initial phases causes the shift to vanish. Conversely, there exists an initial phase that maximizes the shift. In Fig. 1, we plot the shift given by (11) and (12) as a function of the sideband offset $\Omega$ for optimum excitation $b \tau=\pi / 2$ and $\varphi$ chosen for maximum shift at each offset (the final cosine set to one). We see a damped oscillation.

The shift [see (11)] was derived for pure phase modulation, that is, two sidebands having opposite amplitudes, detunings, and initial phases. The shift due to one sideband alone is one-half of (11). Since (12) is invariant when the signs of $b_{1}, \Omega$, and $\varphi$ are all reversed, the contributions of the two sidebands add. Conversely, for a pure amplitude modulation, the second sideband gives a contribution opposite in sign since $b_{1}$ does not change sign. Hence, there is no shift from amplitude modulation. ${ }^{1}$ By comparison, the second-order shift, which is proportional to $b_{1}^{2}$, is insensitive to the sign of $b_{1}$ and cancels for both pure amplitude and pure phase or frequency modulation. Unbalanced sidebands are required for a second-order shift.

\section{B. Ramsey Case}

For Ramsey excitation with two excitation pulses of duration $\tau$ separated by a time $T \gg \tau$, a qualitative picture of the shift can be found by ignoring the phase changes during excitation and considering only the cumulative phase change during the time $T$. The phase modulation causes a phase variation $\Phi(t)=$ $\left(b_{1} / b\right) \sin (\Omega t+\varphi)$. The phase difference between the excitation pulses $\delta \Phi=\Phi(T)-\Phi(0)$ causes a shift $-\delta \Phi$ divided by $T$ [8, Sec. 4.4], [10, Sec. 4.4]. With the aid of a trigonometric identity, we find the shift

$$
\delta \omega_{\mathrm{Ram}}=-\left(2 b_{1} / b T\right) \sin \left(\frac{1}{2} \Omega T\right) \cos \left(\frac{1}{2} \Omega T+\varphi\right) .
$$

This result is valid only for $\Omega \tau \ll 1$, but it shows the rapid oscillation of the shift as a function of $\Omega \mathrm{T}$ and the sinusoidal dependence on $\varphi$. It also gives an estimate of the magnitude of the frequency shift.

To take the phase variation during excitation into account, we utilize the approximate solutions (8) and (9). The transition amplitude for Ramsey excitation is

$$
\beta=\beta^{(2)} \alpha^{(1)} e^{i \Delta T}+\alpha^{(2)} * \beta^{(1)} e^{-i \Delta T}
$$

where the superscripts refer to the first or second excitation. The corresponding transition probability is

$$
P=\left|\alpha^{(1)}\right|^{2}\left|\beta^{(2)}\right|^{2}+\left|\alpha^{(2)}\right|^{2}\left|\beta^{(1)}\right|^{2}+2 \operatorname{Re}\left(\gamma e^{2 i \Delta T}\right)
$$

where $\gamma=\alpha^{(1)} \alpha^{(2)} \beta^{(1) *} \beta^{(2)}$. With slow square-wave modulation, the probability (14) gives a shift

$$
\delta \omega_{\operatorname{Ram}}=-\operatorname{Im} \gamma /(T \operatorname{Re} \gamma)
$$

when the shift is small compared with a Ramsey fringe spacing, and $\gamma$ is evaluated at the modulation amplitude.

For $\tau$ that is much less than $T$, we can neglect detuning during excitation. The approximate solutions [see (8) and (9)] then reduce to

$$
\begin{aligned}
& \alpha_{0}(\tau)=\cos b \tau \\
& \alpha_{1}(\tau)=-i \operatorname{Im} F(\tau) \\
& \beta_{0}(\tau)=-i \sin b \tau \\
& \beta_{1}(\tau)=\operatorname{Re} F(\tau)-i(\Delta / b) \operatorname{Im} F(\tau)
\end{aligned}
$$

\footnotetext{
${ }^{1}$ Coherent amplitude modulation causes a frequency shift not through a static distortion of the lineshape but rather through the demodulation process (or a dynamic distortion of the lineshape). As we have previously described [7], this can be a significant source of error.
} 
with $p$ replaced by $b$ in $F(\tau)$. We assume that the two excitations are identical except for their initial phases. We then find that

$$
\operatorname{Re} \gamma=\sin ^{2} b \tau \cos ^{2} b \tau
$$

where the terms of order $F$ have been neglected. However, the imaginary part of $\gamma$ is of order $F$

$$
\begin{aligned}
\operatorname{Im} \gamma=-\sin ^{2} b \tau \cos b \tau \operatorname{Im} & \left(F^{(1)}+F^{(2)}\right) \\
& -\sin b \tau \cos ^{2} b \tau \operatorname{Re}\left(F^{(1)}-F^{(2)}\right) .
\end{aligned}
$$

The real part of $F(\tau)$ is the same as the imaginary part [see (12)], except that every sine is replaced by cosine and every cosine by sine, with the signs unchanged. If the initial phase for $F^{(1)}$ is $\varphi$, then the initial phase for $F^{(2)}$ is $\varphi+\Omega \tau+$ $\Omega T$. Combining $F^{(1)}$ and $F^{(2)}$ with the aid of trigonometric identities, we find our final result for the first-order sideband shift for the Ramsey excitation to be

$$
\begin{aligned}
\delta \omega_{\text {Ram }}=\frac{4 b_{1} Y(T, \tau)}{(T+\tau h)\left(\Omega^{2}-4 b^{2}\right) \sin 2 b \tau} & \\
& \times \cos \left(\frac{1}{2} \Omega T+\Omega \tau+\varphi\right)
\end{aligned}
$$

where

$$
\begin{aligned}
Y(T, \tau)=2 b \sin 2 b \tau \sin \frac{1}{2} \Omega T-\Omega \cos 2 b \tau \cos \frac{1}{2} \Omega T & \\
+ & \Omega \cos \left(\frac{1}{2} \Omega T+\Omega \tau\right) .
\end{aligned}
$$

When $\Omega=2 b,(16)$ reduces to

$$
\delta \omega_{\text {Ram }}=-\frac{2 b_{1} \tau}{T+\tau h}\left[\begin{array}{r}
\left.\cos b T+\frac{\sin b T}{2 b \tau}+\sin b T \cot 2 b \tau\right] \\
\times \cos (b T+2 b \tau+\varphi) .
\end{array}\right.
$$

When $\Omega$ is small compared with $2 b$ (the sideband lies well within the Rabi width), (16) reduces to

$$
\begin{aligned}
\delta \omega_{\text {Ram }}=-\left(\frac{2 b_{1}}{b(T+\tau h)}\right) \sin & \frac{1}{2} \Omega(T+\tau h) \\
& \times \cos \left[\frac{1}{2} \Omega T+\Omega \tau+\phi\right]
\end{aligned}
$$

where $h=(b \tau)^{-1} \tan b \tau$. The function $h$ gives the same correction to $T$ that defines the separation of the Ramsey fringes for a constant excitation [8]. With the exception of the $\Omega \tau$ phase adjustments, (18) is the same as the approximate result [see (13)].

The dependence on the initial phase in (16) is similar to that of the Rabi shift. Hence, the same comments also apply to the Ramsey shift. Note that (16), like (12), is also an odd function of both $b_{1}$ and $\Omega$. Hence, the Ramsey shift also vanishes for amplitude modulation but not for frequency or phase modulation. ${ }^{1}$

Fig. 2 shows the Ramsey shift as a function of the sideband separation for optimum power and $\varphi$ chosen to make the final cosine equal to unity.

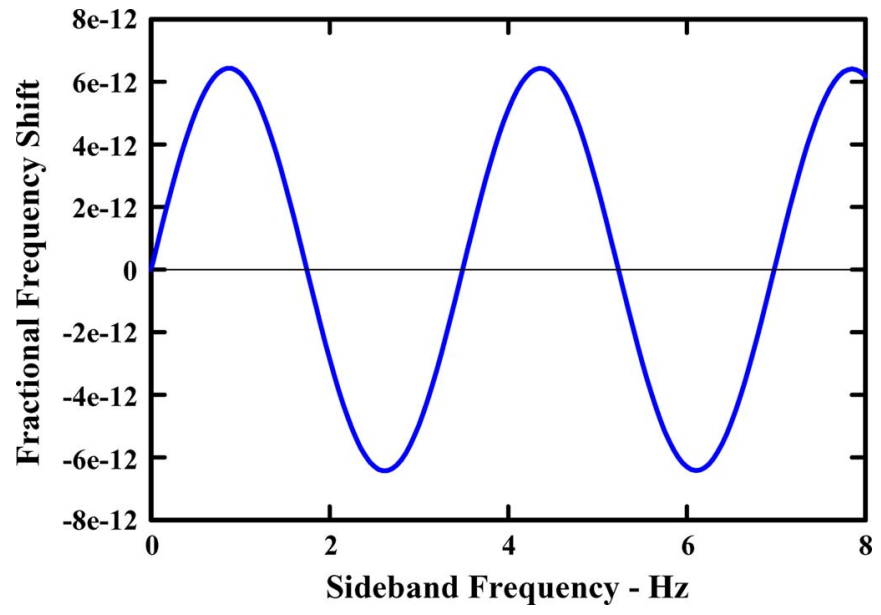

Fig. 2. Theoretical amplitude of the sideband pulling shift for Ramsey excitation with phase modulation. The modulation amplitude was $6^{\circ}$, and the excitation amplitude was optimum.

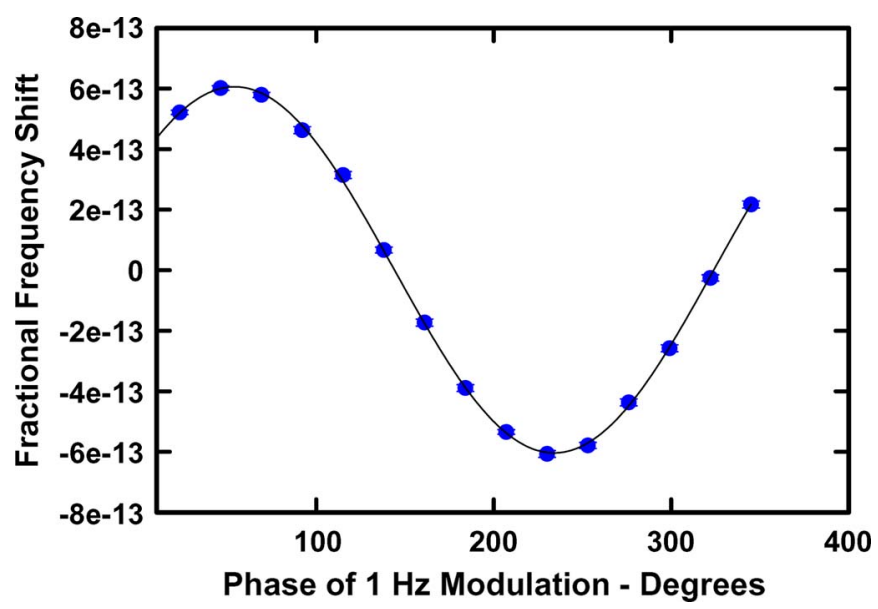

Fig. 3. Initial phase dependence of the sideband pulling shift for Ramsey excitation. The dots are the experimental data, and the solid curve is a fit to the theory. The modulation amplitude was $6^{\circ}$.

\section{EXPERIMENT}

To verify (11) and (16), experiments were performed with the NIST cesium fountain primary frequency standard NIST-F1 [1], [11]. The excitation was phase modulated to allow a large first-order shift with no interfering second-order shift. The modulation was applied at a variable frequency offset and an amplitude of $6^{\circ}(-19.6 \mathrm{dBc})$ relative to the phase of the carrier by feeding the modulation to the direct digital synthesizer that supplies the final tuning of the excitation frequency [12].

Experimentally, it is difficult to know the initial phase of the modulation seen by the atoms. However, when the modulation and the fountain cycling are synchronous, the initial phase can be changed by known amounts by switching the phase of the modulation while the fountain cycling continues uninterrupted. With this technique, we have verified the sinusoidal dependence on the initial phase, as shown in Fig. 3. To find the maximum shift as a function of the initial phase, we make experimental measurements at two initial phases separated by exactly $90^{\circ}$. The root mean square of the two measurements is then independent of $\varphi$ and corresponds to replacing the cosine 


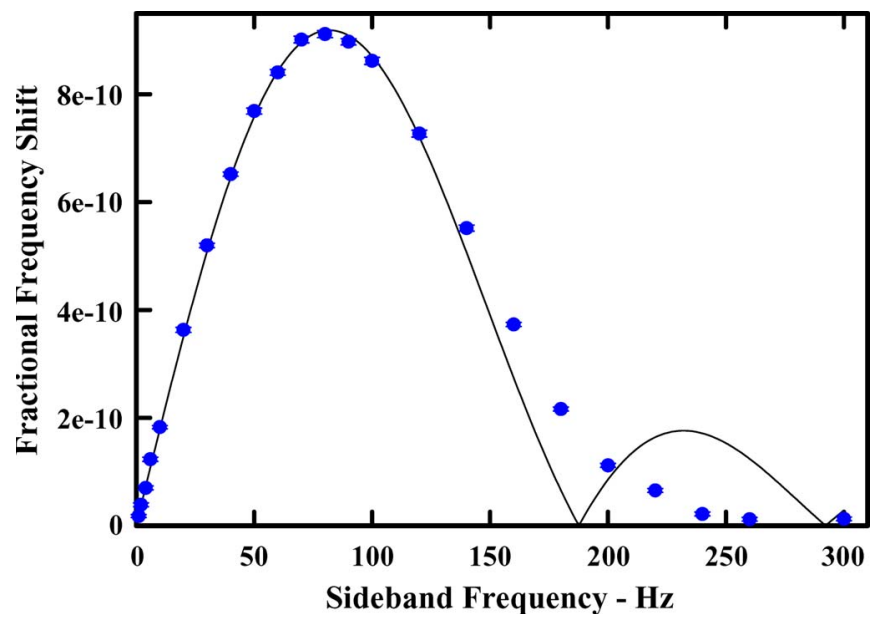

Fig. 4. (Dots) Experimental data and (solid curve) theoretical sideband pulling shifts for Rabi excitation.

containing the initial phase by unity in the theory. Since we always use the positive square root, we are also "rectifying" the shift; therefore, no negative values appear. We repeat these two measurements at each sideband detuning measured. For these measurements, we are careful to preserve the relative phases (coherence) of the modulation source and the fountain measurement cycle.

In Fig. 4, we show some experimental measurements of the Rabi shift along with the "rectified" (absolute value of) theoretical result [see (11)]. In the region beyond $150 \mathrm{~Hz}$, the experimental data deviate from the theory. The likely reason for the disagreement is that the physical extent of the atom cloud is beginning to cause a spread in the initial phases of the sideband seen by the different atoms in the cloud. In NIST-F1, the atom cloud is about $3 \mathrm{~cm}$ high. The atoms at the top of the cloud reach the Ramsey microwave cavity at about $10 \mathrm{~ms}$ before the atoms at the bottom of the cloud. At sideband detunings greater than $50 \mathrm{~Hz}$, the atoms sample a large $(\approx \pi)$ variation in the initial phase of the sideband. Because the cloud has an approximately Gaussian density profile in the vertical direction, this initial phase variation does not eliminate the pulling until the sideband detunings are larger than $250 \mathrm{~Hz}$.

In Fig. 5, we show some sample experimental measurements of the Ramsey shift along with the "rectified" theoretical result [see (16)] for small sideband detuning. No parameter adjustments were made to achieve the fit shown. Fig. 6 shows some older Ramsey shift data [7] at larger sideband detunings. The sideband amplitude was adjusted by $2 \%$, and the Ramsey time $T$ was adjusted by $0.4 \%$ to achieve the fit shown. The Ramsey time adjustment corresponds to a change of $3 \mathrm{~mm}$ in the distance from the initial atom cloud to the cavity. This is less than our uncertainty in the center of mass position of the atom cloud. The amplitude adjustment of $\sim 2 \%$ is within our uncertainty of the optimum power setting for these data. The decrease in amplitude of the rapid oscillations in Fig. 6 when the detuning exceeds the Rabi frequency (about $32 \mathrm{~Hz}$ ) reflects the Rabi envelope of the lineshape. At larger detunings, we anticipate a further decrease like that in Fig. 4 for the same reason discussed there. However, no experimental data have been gathered beyond about $55 \mathrm{~Hz}$. For comparison, the

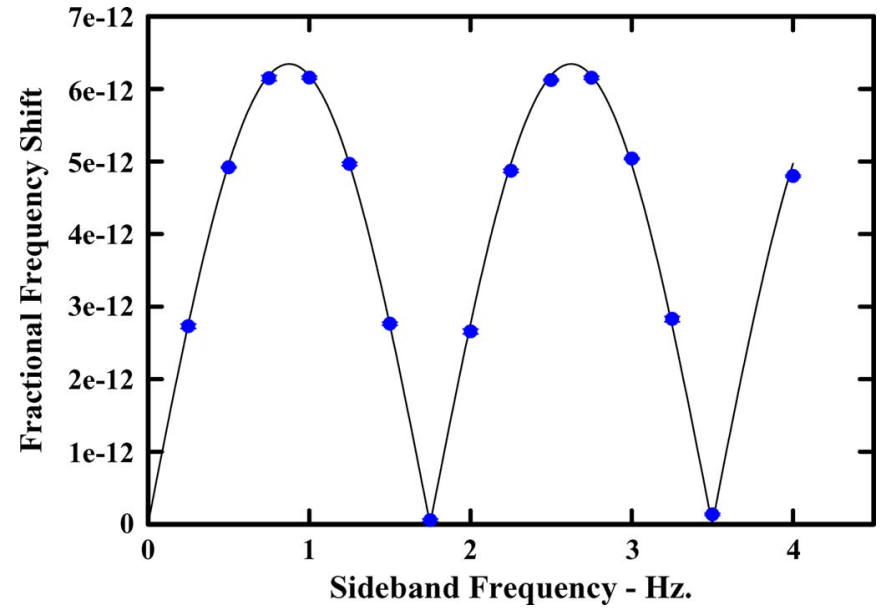

Fig. 5. (Dots) Experimental and (solid curve) theoretical sideband pulling shifts for Ramsey excitation. No adjustable parameters were used, and the modulation depth was $6^{\circ}$.

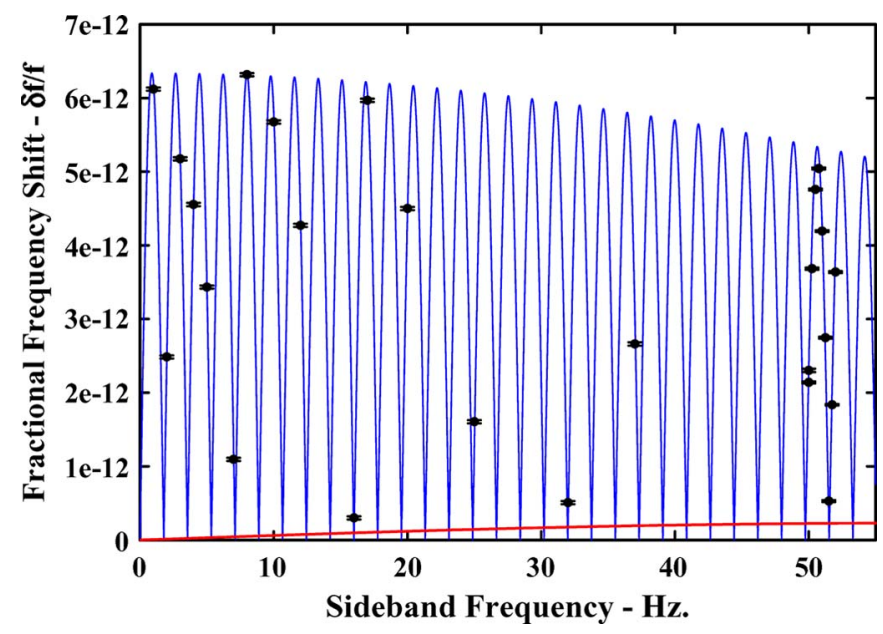

Fig. 6. (Dots) Experimental and (solid curve) theoretical sideband pulling shifts for Ramsey excitation. Slight adjustments of the microwave amplitude $(\sim 2 \%)$ and Ramsey time $(\sim 0.4 \%)$ were made to achieve the fit shown. These adjustments are well within the experimental uncertainties of the microwave amplitude and Ramsey times for these data.

second-order shift (for a single sideband) is shown at the bottom of Fig. 6 on the same scale. The Ramsey oscillations in the second-order shift only occur when the excitation power is not optimum.

\section{CONCLUSION}

The shifts shown in Figs. 5 and 6 are much too large to be tolerated in a primary frequency standard. To ensure that they are not present, one must reduce the amplitude of any sideband until the predicted first-order shift is smaller than the desired uncertainty. One should also choose a measurement cycle period such that any sidebands that might be present have their associated first-order shifts well averaged away after a few tens of cycles. In normal operation of NIST-F1, we set the cycle time near $2 \mathrm{~s}$ but allow it to vary a little over several hours. One must also take care that the instrumental implementation of the measurement cycle introduces no phase changes that are synchronous with the cycle [13]. 


\section{ACKNOWLEDGMENT}

The authors would like to thank F. Levi of INRM for helping us develop an appreciation for these sorts of coherent effects, D.-H. Yu of KRISS for helping us further our understanding of the effects described here, and T. E. Parker, S. Bickman, M. Lombardi, and D. Smith for the many helpful suggestions on the manuscript.

\section{REFERENCES}

[1] S. R. Jefferts, J. Shirley, T. E. Parker, T. P. Heavner, D. M. Meekhof, C. Nelson, F. Levi, G. Constanzo, A. De Marchi, R. Drullinger, L. Hollberg, W. D. Lee, and F. L. Walls, "Accuracy evaluation of NIST-F1," Metrologia, vol. 39, no. 4, pp. 321-336, Dec. 2002.

[2] W. H. Oskay, S. A. Diddams, E. A. Donley, T. Fortier, T. P. Heavner, L. Hollberg, W. M. Itano, S. R. Jefferts, M. J. Jensen, K. Kim, F. Levi, T. E. Parker, and J. C. Bergquist, "Single-atom optical clock with high accuracy," Phys. Rev. Lett., vol. 97, no. 2, pp. 020801-1-020801-4, Jul. 2006.

[3] N. F. Ramsey, "Resonance transitions induced by perturbations at two or more different frequencies," Phys. Rev., vol. 100, no. 4, pp. 1191-1194, Nov. 1955.

[4] J. H. Shirley, "Some causes of resonant frequency shifts in atomic beam machines. I. Shifts due to other frequencies of excitation," J. Appl. Phys., vol. 34, no. 4, pp. 783-788, Apr. 1963.

[5] C. Audoin, M. Jardino, L. S. Cutler, and R. F. Lacy, "Frequency offset due to spectral impurities in cesium-beam frequency standards," IEEE Trans. Instrum. Meas., vol. IM-27, no. 4, pp. 325-329, Dec. 1978.

[6] F. Levi, J. H. Shirley, T. P. Heavner, D.-H. Yu, and S. R. Jefferts, "Power dependence of the frequency bias caused by spurious components in the microwave spectrum in atomic fountains," IEEE Trans. Ultrason., Ferroelectr., Freq. Control, vol. 53, no. 9, pp. 1584-1589, Sep. 2006.

[7] T. P. Heavner, J. H. Shirley, F. Levi, D. Yu, and S. R. Jefferts, "Frequency biases in pulsed atomic fountain frequency standards due to spurious components in the microwave spectrum," in Proc. IEEE Int. Freq. Control Symp., 2006, pp. 273-276.

[8] J. H. Shirley, W. D. Lee, and R. E. Drullinger, "Accuracy evaluation of the primary frequency standard NIST-7," Metrologia, vol. 39, no. 1, pp. 427-458, Feb. 2001.

[9] S. R. Jefferts, J. H. Shirley, N. Ashby, E. A. Burt, and G. J. Dick, "Power dependence of distributed cavity phase-induced frequency biases in atomic fountain frequency standards," IEEE Trans. Ultrason., Ferroelectr., Freq. Control, vol. 52, no. 12, pp. 2314-2321, Dec. 2005.

[10] N. F. Ramsey, Molecular Beams. London, U.K.: Oxford Univ. Press, 1956.

[11] T. P. Heavner, S. R. Jefferts, E. A. Donley, J. H. Shirley, and T. E. Parker, "NIST-F1: Recent improvements and accuracy evaluations," Metrologia, vol. 42, no. 5, pp. 411-422, Oct. 2005.

[12] T. P. Heavner, S. R. Jefferts, E. A. Donley, T. E. Parker, and F. Levi, "A new microwave synthesis chain for the primary frequency standard NIST-F1," in Proc. IEEE Int. Freq. Control Symp., 2005, pp. 308-311.

[13] W. D. Lee, J. H. Shirley, F. L. Walls, and R. E. Drullinger, "Systematic errors in cesium beam frequency standards introduced by digital control of the microwave excitation," in Proc. IEEE Int. Freq. Control Symp., 1995, pp. 113-118.

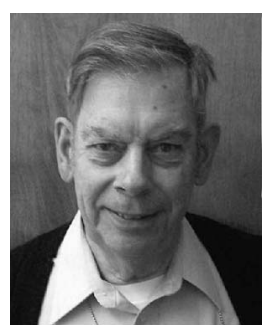

Jon H. Shirley was born in Minneapolis, MN, in 1936. He received the B.A. degree from Middlebury College, Middlebury, VT, in 1957 and the Ph.D. degree from the California Institute of Technology, Pasadena, in 1963.

For many years, he was a Physicist with the National Bureau of Standards, Boulder, CO. He performed theoretical studies on the resonant interaction of radiation with atoms. From 1975 to 1976, he spent a year with the University of Helsinki, Helsinki, Finland. He is currently a Consultant with the Time and Frequency Division, National Institutes of Standards and Technology, Boulder. His principal effort is the determination of biases that affect the U.S. primary frequency standard.

Dr. Shirley received the I. I. Rabi Award at the 2002 IEEE International Frequency Control Symposium.

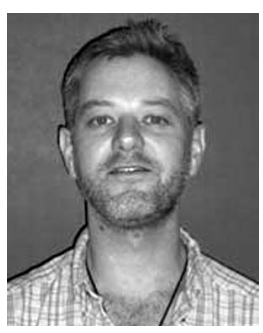

Thomas P. Heavner was born in Alexandria, VA, on October 28, 1966. He received the B.S. degree in physics from the University of Virginia, Charlottesville, in 1989 and the Ph.D. degree from the University of Colorado, Boulder, in 1998. His $\mathrm{Ph} . \mathrm{D}$. dissertation involved the precision mass measurements of single Li ions using a Penning Trap apparatus.

Upon receipt of the Ph.D. degree, he became an NRC Postdoctoral Fellow with the Time and Frequency Division, National Institute of Standards and Technology (NIST), Boulder, where he became a Permanent Staff Member in 2001 with the Atomic Standards Group and where he works on NIST-F1, which is the primary cesium frequency standard for the U.S. government.

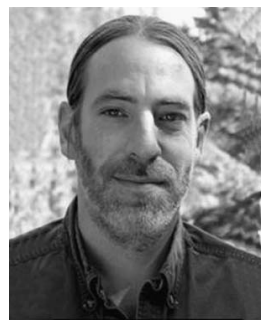

Steven R. Jefferts (M'01) received the B.S. degree in physics from the University of Washington, Seattle, in 1984 and the Ph.D. degree in atomic physics/precision metrology from the University of Colorado, Boulder, 1992. His Ph.D. dissertation involved the ultrahigh-precision mass spectroscopy of light ions.

Since 1992, he has been with the National Institute of Standards and Technology (NIST), Boulder, where he was an NRC Postdoctoral Fellow working on laser-cooled trapped ions. Since 1994, he has been a permanent employee with the Time and Frequency Division, where he leads the research group developing and operating cesium fountain primary frequency standards.

Dr. Jefferts received the Edward Uhler Condon award in 2001, a Department of Commerce Gold Medal in 2004, and the Arthur S. Flemming Award in 2005. He was also the IEEE-UFFC Distinguished Lecturer from 2003 to 2004. 